\title{
Recent progress towards a chiral effective field theory for the NN system
}

\author{
C.J. Yang* and Bingwei Long \\ Dipartimento di Fisica, Universita di Trento, via Sommarive, 14 I-38123 Trento, Italy \\ *E-mail: chieh@science.unitn.it \\ Department of Physics, Sichuan University, 29 Wang-Jiang Road, Chengdu, Sichuan \\ 610064, China \\ E-mail: bingwei@scu.edu.cn
}

\begin{abstract}
Since Weinberg's proposal two decades ago, chiral effective field theory in the $\mathrm{NN}$ sector has been developed and applied up to order $O\left(\left(Q / M_{h i}\right)^{4}\right)$. In principle it could provide a model-independent description of nuclear force from QCD. However, in spite of its huge success, some open issues such as the renormalization group invariance and power counting, still remain to be solved. In this talk we refine the chiral effective field theory approach to the NN system based on a renormalization group analysis. Our results show that a truly model-independent description of NN system can be obtained by a new power counting which treats the subleading order corrections perturbatively.
\end{abstract}

Keywords: Chiral effective field theory, nucleon-nucleon interaction.

\section{Introduction}

Based on the symmetries of Quantum chromodynamics (QCD) in the low energy region $(\leq 1 \mathrm{GeV})$, chiral effective field theory $(\chi \mathrm{EFT})$ enables calculations of strong interaction in the non-perturbative region. However, unlike the pion-pion and pion-nulceon section, where the power countingthe key ingredient which guarantees the intrinsic consistency of an EFTis given clearly from the vertices generated by the chiral Lagrangian, the power counting in nucleon-nucleon $(\mathrm{NN})$ case is hindered by the infrared enhancement and cannot be obtained straightforwardly.

The first step out of the NN problem, as suggested by Weinberg, ${ }^{1}$ is to apply the power counting to the NN potential level first, and then sum the amplitude by iterating the potential in Schrodinger or Lippmann-Schwinger (LS) equation with an ultraviolet cutoff $\Lambda$. Currently, this prescription (the 
so-call Weinberg power counting (WPC)) has been carried out to next-tonext-to-next-to leading order $\left(\mathrm{N}^{3} \mathrm{LO}\right)^{\mathrm{a}}$, and has became the standard of many conventional calculations. ${ }^{2-4}$ However, since Weinberg prescription only applies power counting to the potential level, the systematic control of the theory could be lost in the final amplitude.

\section{Renormalization group analysis}

One way to check whether a proposed scheme is under control is to perform the RG-analysis. RG-analysis carried out at leading order (LO), up to next-to-next-to leading order (NNLO) and $\mathrm{N}^{3} \mathrm{LO}$ based on WPC indicate that the conventional implementation of WPC fails to fulfill the RG requirement once the ultraviolet cutoff of the iteration $\Lambda>1 \mathrm{GeV} .{ }^{5-8}$ Since the chiral expansion is established in powers of $Q / M_{h i}{ }^{\mathrm{b}}$, some authors ${ }^{9}$ have questioned whether the theory is valid once intermediate states have $p \sim \Lambda>M_{h i}$. Thus, it makes little sense to perform RG-analysis for $\Lambda>1$ $\mathrm{GeV}$, even the final on-shell $Q<<M_{h i}$.

A second point of view ${ }^{10-16}$ takes the final amplitude as a partial sum of the (infinitely many) diagrams, then under the assumption that a reasonable separation of scales exists ${ }^{\mathrm{c}}$, in any EFT one should be able to organize those diagrams in a systematic way to absorb the unimportant physics into contact term(s) order by order after a proper renormalization. Thus, as long as $Q<<M_{h i}$, the impact of high-energy physics (which is well-represented by the contact term(s)) in the final amplitude should reduce as the increase of $\Lambda$, since the contribution from physics haven't been integrated out (i.e., from $\Lambda$ to $\infty$ ) becomes smaller and smaller.

The answer of the above in-debating issue actually depends on how the diagrams are organized. It was shown that due to the fine-tuning of low energy constants and a Wigner bond-like effect, ${ }^{17}$ once a cutoff $\Lambda>1 \mathrm{GeV}$ is adopted the renormalization is effectively dominated by one contact term under the WPC scheme. ${ }^{8}$ Moreover, a full-iteration of some type of irreducible two-pion-exchange diagrams could result in a pole-like structure. ${ }^{18}$ Therefore, if one insists to build a NN potential based on $\chi$ EFT and utilizes it later in a conventional way (e.g., inserts it as a potential in Schrodinger

${ }^{a}$ Note that the order here is defined based on the pion-exchange (long-range) part of the potential, which does not necessary equal to the order at the final NN amplitude.

${ }^{\mathrm{b}}$ Here $Q \equiv\left(p, m_{\pi}\right), p$ the NN c.m. momentum, $m_{\pi}$ the pion mass, and the breakdown scale $M_{h i}$ is nominally $m_{\rho} \sim 4 \pi f_{\pi}$.

${ }^{\mathrm{c}}$ In the case where there is no reasonable separation of scales, EFT is impossible. 
or LS equation), he or she needs to stay in $500<\Lambda<1000 \mathrm{MeV}$. The consequence is that a full RG-based analysis becomes inapplicable.

To allow a full RG-analysis, one must give up treating the whole chiral potential non-perturbatively. In other words, for the NN case there exists no "ideal potential" (in the traditional sense) to be extracted or derived. Some parts of the diagrams have to be included perturbatively. Recent works ${ }^{10-13,15,16}$ which treat the subleading chiral potentials in the framework of Distorted-Wave-Born-Approximation enable a full RG- and power counting analysis. Once the $\Lambda$-dependence is under well-control, the estimation of the theoretical error becomes much easier, i.e., the error is given by $O\left(Q^{n+1} / M_{h i}^{n+1}\right)$ up to order-n in the new power counting scheme.

We must point out that the lacks of a RG-analysis cannot rule out the possibility that WPC under the specified range of $\Lambda$ could generate final amplitudes which has the correct power counting, but there is no way to check this so far. On the other hand, a RG-correct scheme could converge too slowly to be useful. Thus, before the full implementation to few- and many-body calculations, one cannot determine the superiority of either scheme. Nevertheless, it is of importance to start with a scheme which allows a full RG-analysis first, then check the power counting step by step to build the theory on a more solid ground.

\section{New Power counting and Future task}

The new power counting developed so far ${ }^{11-13}$ can be summarized as:

1. The LO potential needs to be iterated to all order ${ }^{\mathrm{d}}$, and all subleading chiral potentials are included perturbatively as represented diagrammatically in Fig. 1.

2. The contact terms are determined by RG-analysis to guarantee the correct RG-behavior. As a general rule, for potentials which are singular and attractive at $\mathrm{LO}$ (i.e., $V_{L O}(r \rightarrow 0) \approx-\frac{1}{r^{n}}$ with $n \geq 3$ ), all contact terms need to be promoted one order earlier with respect to WPC.

Phase shifts from the above new scheme are evaluated up to NNLO, and the agreement with the Nijmegen phase-shift analysis ${ }^{19}$ is comparable to those from WPC at the same order.

Furture tasks such as refining power counting with Lepage $\operatorname{plot}^{20}$ or similar techniques, including Delta (1232) contribution, and deciding the power counting for high partial-waves $(l \geq 2)$ are under investigation.

\footnotetext{
$\overline{\mathrm{d}}$ At least for those spin-triplet and $l \leq 1$ partial-waves.
} 


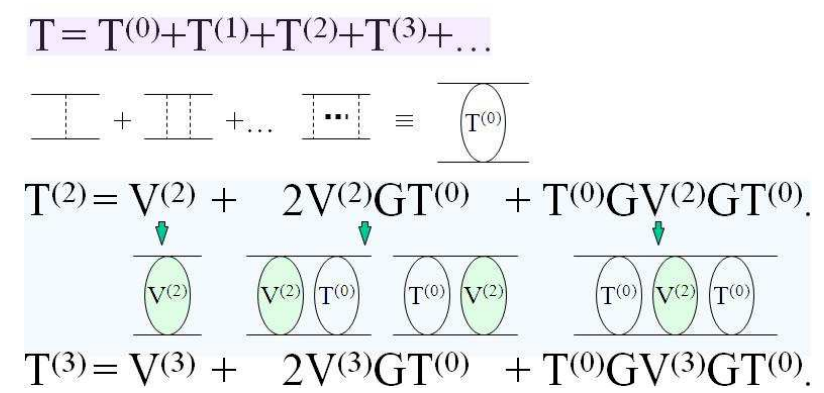

Fig. 1. Diagrammatic representation of the new power counting in the case where the $O(Q)$ contribution is absent. Here $\mathrm{T}, \mathrm{G}$ and $\mathrm{V}$ denotes the T-matrix, propagator and chiral potential. The order of $\mathrm{T}$ and $\mathrm{V}$ is indicated in the superscript.

\section{Acknowledgments}

This work is supported by the US NSF under grant PHYS-0854912, the MIUR grant PRIN-2009TWL3MX and US DOE under contract No. DE-FG02-04ER41338, DE-AC05-06OR2317, E-AC05-06OR23177 and DEFG02-93ER40756.

\section{References}

1. S. Weinberg, Phys. Lett. B 251, 288 (1990); Nucl. Phys. B 363, 3 (1991).

2. D. R. Entem and R. Machleidt, Phys. Rev. C 68, 041001(R) (2003).

3. E. Epelbaum, W. Glöckle and U-G. Meißner, Nucl. Phys. A 747, 362 (2005).

4. E. Epelbaum and U.-G. Meissner, Ann. Rev. Nucl. Part. Sci. 62 159-185 (2012).

5. A. Nogga, R. Timmermans and U. van Kolck, Phys. Rev. C 72054006 (2005).

6. C.-J. Yang, Ch. Elster and D. R. Phillips, Phys. Rev. C 80034002 (2009).

7. C.-J. Yang, Ch. Elster and D. R. Phillips, Phys. Rev. C 80044002 (2009).

8. Ch. Zeoli, R. Machleidt and D. R. Entem, Few-body syst., 54, 12, 2191-2205.

9. E. Epelbaum and G. Gegelia, Eur. Phys. J. A41 341-354 (2009).

10. M. C. Birse, PoS CD 09, 078 (2009) [arXiv:0909.4641 [nucl-th]].

11. Bingwei Long and C.-J. Yang, Phys. Rev. C 84057001 (2011).

12. Bingwei Long and C.-J. Yang, Phys. Rev. C 86024001 (2012).

13. Bingwei Long and C.-J. Yang, Phys. Rev. C 85034002 (2012).

14. Bingwei Long, Phys. Rev. C 88014002 (2013).

15. M. P. Valderrama, Phys. Rev. C 83024003 (2011).

16. M. P. Valderrama, Phys. Rev. C 84064002 (2011).

17. E. P. Wigner, Phys. Rev. 98, 145 (1955).

18. V. Baru et al., Eur. Phys. J. A 4869 (2012).

19. V. G. J. Stoks et al., Phys. Rev. C 48, 792 (1993).

20. G. P. Lepage, arXiv:nucl-th/9706029. 